\title{
Liquid-solid surface phase transformation of fluorinated fullerene on monolayer tungsten diselenide
}

\author{
Zhibo Song, ${ }_{1,2}$ Qixing Wang, ${ }^{1,3}$ Ming-Yang Li ${ }^{4,5}$ Lain-Jong Li, ${ }^{5}$ Yu Jie Zheng, ${ }^{1}$ Zhuo Wang,,${ }^{1,3}$ Tingting Lin, ${ }^{2}$ Dongzhi Chi, ${ }^{2}$ \\ Zijing Ding, ${ }^{1,3, *}$ Yu Li Huang,,${ }^{1,2, \dagger}$ and Andrew Thye Shen Wee ${ }^{1,3, \dagger}$ \\ ${ }^{1}$ Department of Physics, National University of Singapore, 2 Science Drive 3, Singapore 117542, Singapore \\ ${ }^{2}$ Institute of Materials Research \& Engineering (IMRE), A STAR (Agency for Science, Technology, and Research), \\ 2 Fusionopolis Way, Innovis, Singapore 138634, Singapore \\ ${ }^{3}$ Centre for Advanced 2D Materials, National University of Singapore, Block S14, Level 6, 6 Science Drive 2, Singapore 117546, Singapore \\ ${ }^{4}$ Research Center for Applied Sciences, Academia Sinica, Taipei 10617, Taiwan \\ ${ }^{5}$ Physical Sciences and Engineering, King Abdullah University of Science and Technology, Thuwal 23955-6900, Saudi Arabia
}

(Received 8 December 2017; revised manuscript received 13 March 2018; published 4 April 2018)

\begin{abstract}
Hybrid van der Waals heterostructures constructed by the integration of organic molecules and two-dimensional (2D) transition metal dichalcogenide (TMD) materials have useful tunable properties for flexible electronic devices. Due to the chemically inert and atomically smooth nature of the TMD surface, well-defined crystalline organic films form atomically sharp interfaces facilitating optimal device performance. Here, the surface phase transformation of the supramolecular packing structure of fluorinated fullerene $\left(\mathrm{C}_{60} \mathrm{~F}_{48}\right)$ on single-layer tungsten diselenide $\left(\mathrm{WSe}_{2}\right.$ ) is revealed by low-temperature scanning tunneling microscopy, from thermally stable liquid to solid phases as the coverage increases. Statistical analysis of the intermolecular interaction potential reveals that the repulsive dipole-dipole interaction induced by interfacial charge transfer and substrate-mediated interactions play important roles in stabilizing the liquid $\mathrm{C}_{60} \mathrm{~F}_{48}$ phases. Theoretical calculations further suggest that the dipole moment per $\mathrm{C}_{60} \mathrm{~F}_{48}$ molecule varies with the surface molecule density, and the liquid-solid transformation could be understood from the perspective of the thermodynamic free energy for open systems. This study offers insights into the growth behavior at 2D organic/TMD hybrid heterointerfaces.
\end{abstract}

DOI: 10.1103/PhysRevB.97.134102

\section{INTRODUCTION}

The integration of two-dimensional (2D) materials with organic semiconductors to create layered assemblies with unique properties for flexible electronic devices has attracted increasing interest recently [1-7]. Promising device performances have been demonstrated in field-effect transistors and optoelectronics devices based on such organic-2D heterojunctions [4-6]. For instance, p-n heterojunctions have been fabricated with the integration of a p-type molecule (e.g., pentacene) and a $2 \mathrm{D}$ n-type semiconductor, $\mathrm{MoS}_{2}$, demonstrating good photovoltaic properties [8-11]. The deposition of selected organic acceptor/donor molecules on 2D materials is also a promising noninvasive doping approach for tuning the majority charge carrier type and manipulating the transport barrier in 2D layered composites according to the application requirements [12-14]. The interfacial properties at the organic-2D heterointerfaces are complex and need to be fully understood in order to design and construct a wide range of organic-2D hybrid structures with desirable properties.

2D layered materials have atomically smooth surfaces with the absence of dangling bonds. Organic molecules are largely bound by weak surface van der Waals (vdW) forces, and form high-quality crystalline organic films, facilitating good

\footnotetext{
*Corresponding author: c2ddizi@ nus.edu.sg

${ }^{\dagger}$ Corresponding author: huangyl@imre.a-star.edu.sg

${ }^{\ddagger}$ Corresponding author: phyweets@nus.edu.sg
}

device performance $[5,9,15]$. The situation is complicated by the existence of multiple substrate-mediated interactions. The surfaces of 2D crystals can modulate the growth of adsorbate layers although they are chemically inert. For example, moiré patterns induced by the underlying substrate on the otherwise homogeneous 2D materials can be used as a nanoscale template to selectively adsorb atoms, molecules, and nanoclusters at specific adsorption sites [16-20]. Repulsive dipole-dipole interactions induced by charge transfer (CT) are another class of interfacial interactions that have been intensively studied for different adsorbates on metallic substrates [21-24]. As a result of the subtle competition between vdW forces and multiple substrate-mediated interactions, surface phase transformations, e.g., from disordered loose-packed conformations to well-ordered close-packed structures, have been observed during growth [22,25-27]. Recent investigations of graphene on supported substrates revealed that the adsorbate surface reconstruction can be also tuned by $\mathrm{CT}$ across the heterointerfaces $[12,28,29]$. In particular, the self-assembly of organic adsorbates on layered and 2D transition metal dichalcogenides (TMDs) will be an important model system for understanding such hybrid heterointerfaces [30,31].

In this report, we explore the phase transformation of fluorinated fullerene $\left(\mathrm{C}_{60} \mathrm{~F}_{48}\right)$ on single-layer tungsten diselenide (SL-WSe ${ }_{2}$ ) on a graphite substrate [Fig. 1(a)] using low-temperature (LT) scanning tunneling microscopy (STM) supported by first-principles calculations. $\mathrm{C}_{60} \mathrm{~F}_{48}$ is selected as a model molecule because it is a molecular acceptor [32] with applications in lithium batteries [33,34], electronic devices 

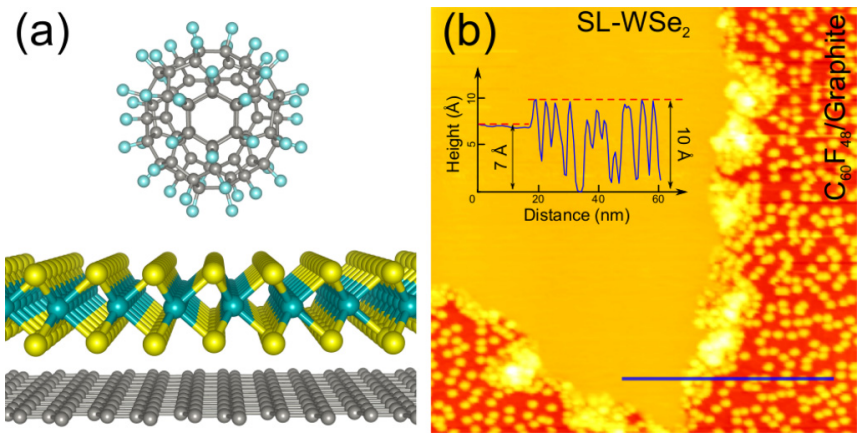

FIG. 1. $\mathrm{C}_{60} \mathrm{~F}_{48}$ molecules on SL-WSe 2 /graphite. (a) A schematic model of the $\mathrm{C}_{60} \mathrm{~F}_{48} / \mathrm{SL}-\mathrm{WSe}_{2} /$ graphite heterostructure (gray: carbon; turquoise: fluorine; yellow: selenium; cyan: tungsten). (b) A large-scale STM image shows a typical triangular $\mathrm{SL}_{-} \mathrm{WSe}_{2}$ island on the graphite substrate with a very low coverage of $\mathrm{C}_{60} \mathrm{~F}_{48}$ molecules $(<0.01 \mathrm{ML})\left(V_{\text {tip }}=-3.1 \mathrm{~V}\right.$; image size: $\left.120 \times 120 \mathrm{~nm}^{2}\right)$. The inset lateral profile corresponding to the blue line reveals a height of $7 \AA$ for $\mathrm{SL}-\mathrm{WSe}_{2}$ and $10 \AA$ for $\mathrm{C}_{60} \mathrm{~F}_{48}$ molecules.

[35-37], and so on [38,39]. $\mathrm{WSe}_{2}$ represents as a prototypical 2D-TMD semiconductor with a high photon quantum yield $[30,31,40,41]$. As revealed by STM investigations, a liquidsolid surface phase transition is observed from a loose-packed liquid phase at dilute coverage to a close-packed solid phase at high coverage. Through careful statistical analyses, we reveal that $\mathrm{vdW}$ forces, dipole-dipole interactions, and substratemediated interactions play important roles in driving the phase transformation. Theoretical calculations based on density functional theory (DFT) further confirm that the repulsive dipole-dipole interactions are induced by CT from substrate to molecules and vary with the surface molecular density. These observations offer insights into the understanding of the interfacial interactions governing organic molecules physisorbed on 2D semiconducting TMD surfaces.

\section{METHODS}

SL-WSe ${ }_{2}$ was directly grown on a graphite substrate by the chemical vapor deposition (CVD) method using $\mathrm{WO}_{3}$ and Se powders as precursors [42]. Before STM measurements, the $\mathrm{SL}-\mathrm{WSe}{ }_{2}$ sample was degassed in a preparation chamber overnight at $400{ }^{\circ} \mathrm{C}$ with base pressure better than $10^{-9}$ mbar. A $\sim 10 \%$ overall coverage of $\mathrm{SL}-\mathrm{WSe}_{2}$ was verified by statistical analysis over the graphite surface. $\mathrm{C}_{60} \mathrm{~F}_{48}$ (nano-C, 99.6\%) was thermally deposited onto the $\mathrm{WSe}_{2}$ /graphite and bare graphite substrates held at room temperature. The deposition rate is about $0.04 \mathrm{ML}$ per minute, where $1 \mathrm{ML}$ is defined as one full close-packed monolayer $\mathrm{C}_{60} \mathrm{~F}_{48}$. The overall coverages were further calibrated by taking statistical over large-scale STM images. After deposition, samples were transferred in situ from the deposition chamber $\left(\sim 10^{-9}\right.$ mbar $)$ to the attached STM chamber $\left(\sim 10^{-10}\right.$ mbar $)$ for subsequent STM measurements.

All STM measurements were carried out in a highresolution Omicron LT-STM interfaced to a Nanonis controller at $77 \mathrm{~K}$, using an electrochemically etched tungsten tip under a constant current mode. The sample holder was grounded and bias voltages were applied on the tip.
The first-principles calculations were carried out using the Vienna $a b$ initio simulation package code [43], employing projector augmented wave pseudopotentials [44,45] and the Perdew-Burke-Ernzerholf form [46] of the exchangecorrelation functional. To simulate different molecular coverage, supercells with different sizes were built to contain a $\mathrm{C}_{60} \mathrm{~F}_{48}$ molecule: $(4 \times 4) \mathrm{WSe}_{2}$ on $(2 \sqrt{7} \times 2 \sqrt{7})$ graphene (close to the solid phase), $(3 \sqrt{3} \times 3 \sqrt{3}) \mathrm{WSe}_{2}$ on $(7 \times 7)$ graphene, $(\sqrt{57} \times \sqrt{57}) \mathrm{WSe}_{2}$ on $(11 \times 11)$ graphene, and $(6 \sqrt{3} \times 6 \sqrt{3}) \mathrm{WSe}_{2}$ on $(14 \times 14)$ graphene (close to the liquid phase). Here, the graphite lattice constant of $2.46 \AA$ obtained from experiment was used, and the $\mathrm{SL}-\mathrm{WSe}_{2}$ supercells on a single-layer graphene were optimized using the vdW density functional method (optB88-vdW) [47,48]. A vacuum of $1.7 \mathrm{~nm}$ along the $z$ direction is included to meet periodic boundary conditions. For comparison, $\mathrm{C}_{60} \mathrm{~F}_{48}$ molecules adsorbed on bare graphene substrate were also calculated (see the Supplemental Material [49]). The energy cutoff for plane waves was set to be $400 \mathrm{eV}$, and the criterion for total energy convergence was set to $10^{-4} \mathrm{eV}$. During geometry optimization, all atoms were relaxed until the magnitude of forces was less than $0.01 \mathrm{eV} / \AA$.

\section{RESULTS AND DISCUSSION}

Figure 1(a) shows a schematic structure of $\mathrm{C}_{60} \mathrm{~F}_{48}$ adsorbed on a SL-WSe ${ }_{2}$ on a graphite substrate. The $\mathrm{SL}_{-\mathrm{WSe}} /$ graphite sample was grown by the CVD method, and the SL-WSe flakes can be easily distinguished by their typical triangle shapes with lateral sizes over hundreds of nm on the graphite surface [30,40]. Figure 1(b) demonstrates a large-scale STM image $\left(120 \times 120 \mathrm{~nm}^{2}\right)$ of the SL-WSe $2 /$ graphite surface with a very low coverage of $\mathrm{C}_{60} \mathrm{~F}_{48}$ (less than $0.01 \mathrm{ML}$ ). Bright balls observed in the STM image are attributed to the spherical $\mathrm{C}_{60} \mathrm{~F}_{48}$ molecules, which are exclusively adsorbed on the graphite surface (right side and left lower corner) leaving the $\mathrm{WSe}_{2}$ surface empty. A line profile corresponding to the blue solid line in Fig. 1(b) crossing over the bare $\mathrm{WSe}_{2}$ surface and the $\mathrm{C}_{60} \mathrm{~F}_{48}$ molecules is shown in the inset. It confirms a thickness of $7 \AA$ for the $\mathrm{WSe}_{2}$ monolayer in the left side, and a height of $10 \AA$ for a single $\mathrm{C}_{60} \mathrm{~F}_{48}$ layer at the right side. The individual molecules are loosely adsorbed on the graphite surface with intermolecular distances of over several $\mathrm{nm}$. The bare graphite surface is observable at the same time (Fig. S1 of the Supplemental Material [49]). Detailed analyses will be discussed later. Furthermore, the $\mathrm{C}_{60} \mathrm{~F}_{48}$ molecules also prefer to attach to the $\mathrm{WSe}_{2}$ edges due to the relatively high chemical reactivity. As the molecular coverage increases, $\mathrm{C}_{60} \mathrm{~F}_{48}$ molecules start to adsorb in a close-packed arrangement on the $\mathrm{WSe}_{2}$ surface before the graphite surface is fully covered ( Fig. S1).

Figure 2 reveals the phase transformation on the $\mathrm{SL}-\mathrm{WSe}_{2}$ surface as the $\mathrm{C}_{60} \mathrm{~F}_{48}$ coverage gradually increases from $\sim 0.01$ $\mathrm{ML}$ to $0.5 \mathrm{ML}$. When the overall coverage is $0.01 \mathrm{ML}$, the SL-WSe 2 surface is partially decorated by sparse $\mathrm{C}_{60} \mathrm{~F}_{48}$ clusters in Fig. 2(a), where each cluster is composed of less than 10 molecules, and the intercluster spacing is larger than $5 \mathrm{~nm}$. As the coverage increases slightly to $0.03 \mathrm{ML}$, small islands comprising over 10 isolated molecules are found in Fig. 2(b). These small islands with lateral sizes of tens of 

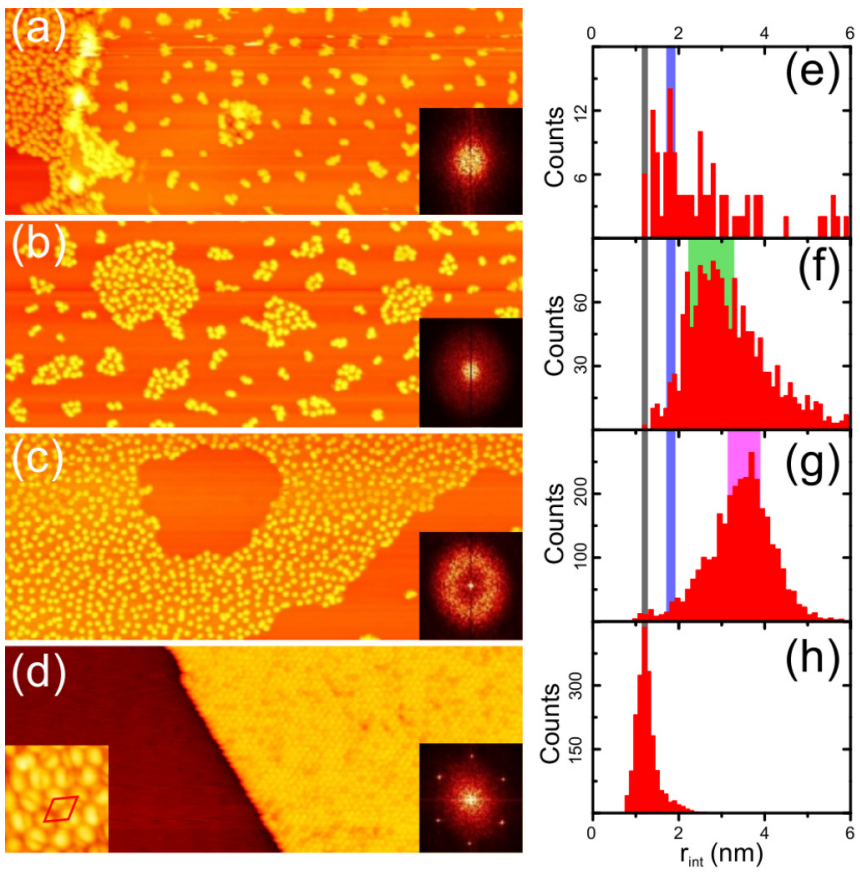

FIG. 2. Liquid-solid surface phase transformation of $\mathrm{C}_{60} \mathrm{~F}_{48}$ on SL-WSe ${ }_{2}$ with the increasing coverage. (a)-(d) STM images reveal the different conformations of $\mathrm{C}_{60} \mathrm{~F}_{48}$ on SL-WSe $\mathrm{S}_{2}$ with the coverages of $\sim 0.01 \mathrm{ML}, 0.03 \mathrm{ML}, 0.05 \mathrm{ML}$, and $0.5 \mathrm{ML}$, respectively, and the insets in the lower right corners show the corresponding FFT images [image size: (a)-(c) $86 \times 215 \mathrm{~nm}^{2}$, (d) $40 \times 100 \mathrm{~nm}^{2} ; V_{\text {tip }}=-3.1 \mathrm{~V}$ ]. Left lower edge in panel (a) is $\mathrm{C}_{60} \mathrm{~F}_{48}$ on bare graphite. The inset in the lower left corner in (d) shows a submolecular-resolution STM image of the close-packed $\mathrm{C}_{60} \mathrm{~F}_{48}$ island $\left(6 \times 6 \mathrm{~nm}^{2} ; V_{\text {tip }}=-3.1 \mathrm{~V}\right)$. (e)(h) Histograms of the three nearest molecule-molecule separations correspond to (a)-(d), respectively, where the colored stripes highlight the different peak positions for different coverages.

$\mathrm{nm}$ are identified as a "droplet-like" phase. Small molecular clusters are still observable but decrease in density, indicating that no new nucleation cores form. When the coverage further increases to 0.05 ML in Fig. 2(c), large loose-packed islands extending over hundreds of $\mathrm{nm}$ form with the merging of small $\mathrm{C}_{60} \mathrm{~F}_{48}$ clusters and islands. In the dilute coverages $(0.01,0.03$, and $0.05 \mathrm{ML}$ ), it is worth noting that almost every $\mathrm{C}_{60} \mathrm{~F}_{48}$ molecule is isolated from each other with intermolecular separations much larger than its $\mathrm{vdW}$ diameter $(\sim 1 \mathrm{~nm})$. Finally, a close-packed phase is found when the coverage is higher than 0.1 ML. Figure 2(d) shows a solid $\mathrm{C}_{60} \mathrm{~F}_{48}$ island coexisting with the uncovered $\mathrm{WSe}_{2}$ surface (left side) at 0.5 ML. From the close-up $\left(6 \times 6 \mathrm{~nm}^{2}\right)$ in Fig. $2(\mathrm{~d})$, we can see that the molecular arrays are closely packed and well aligned. The $\mathrm{C}_{60} \mathrm{~F}_{48}$ unit cell highlighted by a red rhombus has a lattice constant of $1.22 \pm 0.04 \mathrm{~nm}$, consistent with the unit cell size of the fcc (111) plane of the $\mathrm{C}_{60} \mathrm{~F}_{48}$ crystal [50].

The coverage-dependent phase transformation is also reflected in the corresponding fast Fourier transform (FFT) images generated by WSxM software [51]. Bright diffuse disks are obtained in the insets of Figs. 2(a) and 2(b) due to the small disordered molecular clusters and islands, respectively. A blurred ring is generated in the Fig. 2(c) inset, indicating a certain distribution of intermolecular separations for the $\mathrm{C}_{60} \mathrm{~F}_{48}$ molecules in the loose-packed molecular islands, which is a possible liquid phase. Finally, a hexagonal pattern comprising six sharp spots is obtained in the FFT image of the Fig. 2(d) inset, reflecting the formation of the close-packed well-ordered hexagonal structure above a critical coverage. A similar phase transformation is also observed on the bare graphite surface (Fig. S4 of the Supplemental Material [49]).

Histograms of the intermolecular separations are demonstrated in Figs. 2(e)-2(h). Here, only the three nearest molecule-molecule separations $\left(r_{\text {int }}\right.$, from center to center) were extracted from the STM images for all coverages [22]. The technique used to extract intermolecular separations is provided in Fig. S2 of the Supplemental Material [49]. Obviously, the distributions of the $\mathrm{C}_{60} \mathrm{~F}_{48}$ molecules on the SL-WSe surface are not random (Fig. S3) [52]. At the coverage of 0.01 ML, a peak observed at $1.8 \mathrm{~nm}$ is highlighted by a blue stripe in Fig. 2(e). In Fig. 2(f), the counts at $r_{\text {int }}>2 \mathrm{~nm}$ increase significantly and a strong peak at $\sim 2.8 \mathrm{~nm}$ (green stripe) appears, arising from the small droplet-like islands formed at $0.03 \mathrm{ML} \mathrm{C}_{60} \mathrm{~F}_{48}$. The peak at $\sim 1.8 \mathrm{~nm}$ (blue stripe) is still visible due to the coexisting molecular clusters, as revealed in Fig. 2(b). As the coverage increases to $0.05 \mathrm{ML}$, a significant peak at $\sim 3.7 \mathrm{~nm}$ (purple stripe) is obtained in Fig. 2(g) corresponding to a possible liquid phase [Fig. 2(c)], while the peak at $1.8 \mathrm{~nm}$ almost disappears. At last, a sharp peak at $1.2 \mathrm{~nm}$ is observed in Fig. 2(h), consistent with the close-packed supramolecular structure demonstrated in Fig. 2(d). A lower cutoff at $1.2 \mathrm{~nm}$ (gray stripe) is observed in Figs. 2(e)-2(g) for the three sparse phases, attributed to the solid phase limit.

Another method to identify the liquid or solid nature of a system is the utilization of the radial distribution function (RDF) [53] to estimate the probability of finding a molecule at a distance of $r$ away from a given reference molecule. The RDF probability is determined by

$$
g(r)=\frac{1}{N^{2}} \sum_{i=1}^{N} \sum_{j=1}^{N}\left\langle\delta\left(\left|\mathbf{r}_{i j}\right|-r\right)\right\rangle,
$$

where $r_{i j}$ is the intermolecular separation between the $i$ th and $j$ th molecules. Figures 3(a) and 3(b) provide the computed RDF curves for 0.05 ML [Fig. 2(c)] and 0.5 ML [Fig. 2(d)] $\mathrm{C}_{60} \mathrm{~F}_{48}$ on SL-WSe 2 , respectively, after recalibration (see the Supplemental Material [49]). The nearest-neighbor distances from more than 2000 molecules were counted for each plot. In Fig. 3(a), $g(r)$ shows a peak at $3.9 \mathrm{~nm}$ corresponding to the distance with the highest probability of finding a nearestneighbor molecule, which is consistent with the peak observed at the molecular distribution histogram in Fig. 2(g). The second peak appearing at $7.9 \mathrm{~nm}$ is twice the distance of the first peak. At larger distances, the probability approaches to a constant of around $0.065 \mathrm{~nm}^{-2}$, which corresponds to the molecular density in the loose-packed liquid-like phase. An RDF curve for an ideal Lennard-Jones liquid on a surface is drawn as a black dashed line for comparison in Fig. 3(a). Indeed, the RDF curves obtained from our STM experiment (solid) and the ideal liquid (dashed) show agreements in the first and second coordination shells, confirming that the loose-packed conformation observed at $0.05 \mathrm{ML}_{60} \mathrm{~F}_{48}$ on SL-WSe 2 [Fig. 2(c)] is indeed a liquid phase. The liquid $\mathrm{C}_{60} \mathrm{~F}_{48}$ phase is less ordered than the ideal liquid, lacking long-range 

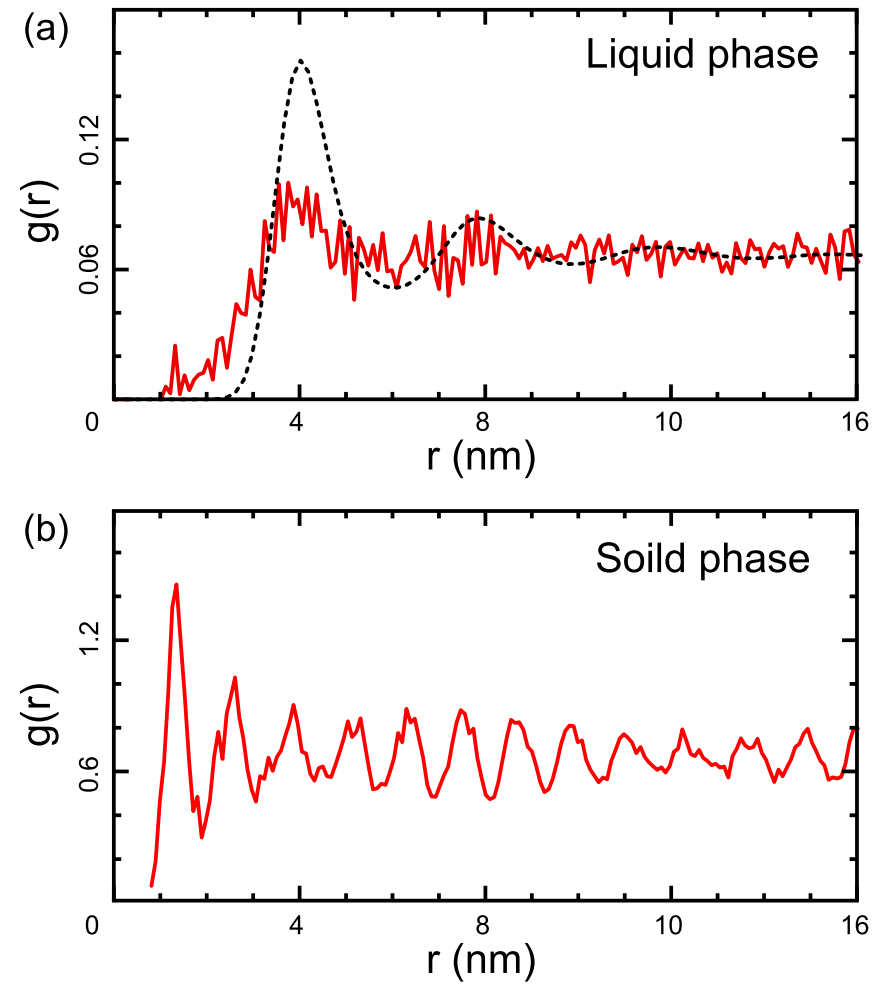

FIG. 3. Radial distribution function $g(r)$ of the liquid and solid $\mathrm{C}_{60} \mathrm{~F}_{48}$ phases on $\mathrm{SL}_{-} \mathrm{WSe}_{2}$. (a) Comparison of the $g(r)$ distribution for the liquid phase obtained from STM data (red solid line) with an ideal Lennard-Jones liquid (black dashed line) reveals a good agreement. (b) The $g(r)$ distribution for the close-packed structure shows a typical oscillating characteristic for a solid phase. $g(r)$ is in units of $\mathrm{nm}^{-2}$. Here, the molecular positions and intermolecular distances have been carefully recalibrated based on the fitted eccentricity of ellipse-shaped FFT images and the threefold symmetry of the $\mathrm{WSe}_{2}$ lattices (see the Supplemental Material [49]).

order as the experimental RDF curve becomes constant shortly after the second coordination shell. A minor peak at $\sim 1.3 \mathrm{~nm}$ corresponding to the limit by the solid phase is observable. In Fig. 3(b), the RDF plot for the $0.5 \mathrm{ML} \mathrm{C}_{60} \mathrm{~F}_{48}$ demonstrates a typical oscillating characteristic for a solid single-crystalline phase [53]. Clearly, the oscillating sharp peaks with a basic periodicity of $\sim 1.3 \mathrm{~nm}$ originate from the lattice constant of the well-ordered close-packed islands shown in Fig. 2(d). At larger distance, this probability curve oscillates at around $0.7 \mathrm{~nm}^{-2}$ corresponding to the molecular density of the solid phase $\mathrm{C}_{60} \mathrm{~F}_{48}$ on SL-WSe 2 . Therefore, with the combination of STM measurements and RDF plots, we have revealed the formation of $\mathrm{C}_{60} \mathrm{~F}_{48}$ liquid and solid phases on $\mathrm{SL}_{-\mathrm{WSe}}$ surface at different coverages with good thermal stability at $77 \mathrm{~K}$.

The formation of the surface liquid phase for $\mathrm{C}_{60} \mathrm{~F}_{48}$ on $\mathrm{SL}_{-} \mathrm{WSe}_{2}$ is interesting due to the absence of strong molecule-substrate coupling with the chemically inert substrate at each nonpolar spherical molecule. It is unlikely that the molecules are anchored at specific adsorption sites on such substrates, although moiré patterns are observable on the $\mathrm{SL}-\mathrm{WSe}_{2} /$ graphite $[31,40]$. The low corrugation of interaction potential in the $\mathrm{WSe}_{2} /$ graphite surface is also one of the reasons for the observed diffusing $\mathrm{C}_{60} \mathrm{~F}_{48}$ molecules

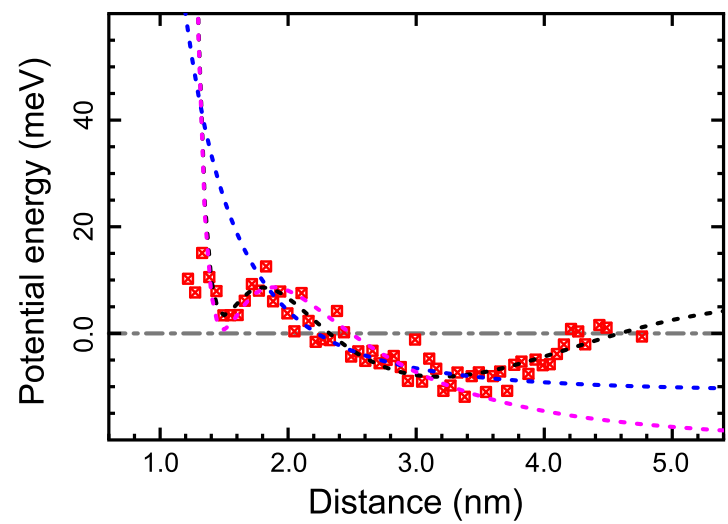

FIG. 4. Evaluation of the intermolecular potential energy $E(r)$ for $\mathrm{C}_{60} \mathrm{~F}_{48}$ liquid phase. $E(r)$ marked by red squares is evaluated from the histogram of the nearest-neighbor separation based on STM data. The dashed curves show the asymptotic fitting by considering dipoledipole interactions only (blue); dipole-dipole interactions plus vdW interaction (pink); and vdW interaction, dipole-dipole interaction, and surface-state-mediated interactions (black). A dipole moment of $3.6 e \AA$ for the molecule is derived for the black dashed curve.

[54-56]. Two other reasons for the observed high mobility of the molecules are tip-induced mobility and relatively weak molecule-substrate interactions which are evident by the easy desorption of molecules at low annealing temperature $\left(100^{\circ} \mathrm{C}\right)$. In the loose-packed phases (at lower coverages), repulsive lateral interactions must be present; otherwise the molecules would aggregate into close-packed islands with the attractive vdW forces. The most likely source of the repulsive forces is the intermolecular dipole-dipole interactions induced by interfacial $\mathrm{CT}$ for the nonpolar $\mathrm{C}_{60} \mathrm{~F}_{48}$ molecules, which has been observed for other atoms/molecules adsorbed on metallic substrates [57,58]. Interfacial dipoles induced by CT has been demonstrated for a solid $\mathrm{C}_{60} \mathrm{~F}_{48}$ layer on top of a SL-WSe 2 in our previous study [31].

To clarify the nature of the repulsive interactions in the liquid phase, we evaluate the $\mathrm{C}_{60} \mathrm{~F}_{48}$ intermolecular interaction potential $E(r)$ quantitatively. In terms of two-body interactions, $E(r)$ can be given by

$$
E(r)=-k_{B} T \ln \left[\frac{f(r)}{f_{\mathrm{ran}}(r)}\right],
$$

where $f(r)$ is the probability distribution histogram of the nearest-neighboring (NN) molecular separations extracted from the STM image, and $f_{\text {ran }}(r)$ is the random separation distribution for noninteracting adsorbates [52,59,60]. The histogram of the NN molecular separations for the liquid phase is given in Fig. S3 of the Supplemental Material [49], and it is slightly different from Fig. 2(g), which was extracted from the three nearest intermolecular separations.

As demonstrated in Fig. 4, the extracted $E(r)$ (red squares) does not show a monotonic behavior; rather, local minima are observed. The valley at $r=3.5 \mathrm{~nm}$ with the lowest potential energy is consistent with the main peak at $r=$ $3.7 \mathrm{~nm}$ in Fig. 2(c), that is, the most energetically favorable intermolecular separation. The small difference in the distance could be attributed to the different statistical method used. The local minimum at $1.4 \mathrm{~nm}$ gives rise to the observed 
limits in Fig. 2(c) and in Fig. 3(a), corresponding to the lattice constant in the well-defined solid phase. Any deviation from the minimum points would cause an increase in potential energy. Such oscillation behavior in the potential $E(r)$ cannot be explained by vdW forces only (Fig. 4, blue dashed curve). With the addition of repulsive dipole-dipole interactions, which decays as $1 / r^{3}$, the fitting agrees well with the experimental $E(r)$ at small distances, but fails at large distances with a monotonic decreasing behavior (Fig. 4, pink dashed curve, and Fig. S9 of the Supplemental Material [49]). Therefore, substrate-mediated interactions through the adsorbate-induced electronic perturbation of surface potential should be considered [61]. That is, the surface potential can be modulated by adsorbates/defects acting as scattering centers for surface electrons (e.g., Friedel oscillations [62]). Upon adsorption of another particle, the final adsorption sites of the adsorbates are determined by the (interference) interactions between the induced perturbations of both particles. Previous exploitations on the substrate-mediated interactions as a means to control the surface self-assemblies have been mainly focused on noble metals $[52,59,60]$ and graphene $[20,57]$ substrates. Observations of Friedel oscillations induced by atomic-scale defects have been previously reported in graphene [63-66] and monolayer and bilayer $\mathrm{WSe}_{2}[67,68]$ (see the Supplemental Material [49]). Therefore, we can assume that the substratemediated interactions are significant, and the same perturbation mechanism is applicable to any substrate, whether noble metals or 2D TMD semiconductors.

Based on the theory of Hyldgaard and Persson [52,69], the substrate-mediated potential is a function of the distance $r$ (see the Supplemental Material [49]),

$$
E_{\text {sub }} \propto-\frac{\sin (2 A r+B)}{r^{2}},
$$

where $A$ is a parameter associated with the scattering wave vector.

By considering all three components, vdW forces (LennardJones potential) $E_{L J}$, dipole-dipole interaction $E_{\text {dipole }}$, and substrate-mediated interactions $E_{\text {sub }}$, the total potential can be written as

$$
E(r)=E_{L J}+E_{\text {dipole }}+E_{\text {sub }} .
$$

As shown in Fig. 4, the best fitting (black dashed curve) is obtained when $A$ is equal to $0.6 \pm 0.2 \mathrm{~nm}^{-1}$. The good fit to the experimental data indicates the relevance of formula (3) to the $\mathrm{WSe}_{2}$ system (and possibly other 2D semiconductors). However, it is difficult to determine the momentum corresponding to parameter $A$, because of the multiple scattering channels in $\mathrm{WSe}_{2}$ [67,68]. Possible origins include the intravalley scattering occurring at the $K$ point [67] and possible interfacial electronic states [70] formed at the $\mathrm{WSe}_{2}$-graphite heterointerface, and further theoretical and experimental studies are required (see the Supplemental Material [49]).

From the final fitting (black dashed curve in Fig. 4), $\mu$ is derived to be $3.6 e \AA$ for the liquid $\mathrm{C}_{60} \mathrm{~F}_{48}$ on $\mathrm{SL}_{-} \mathrm{WSe}_{2}$. DFT calculations were carried out to estimate the dipole moment $\mu$ of $\mathrm{C}_{60} \mathrm{~F}_{48}$ and its origins. As discussed in the Supplemental Material [49] and our previous paper [31], the dipole originates from charge transfer from the substrate to the $\mathrm{C}_{60} \mathrm{~F}_{48}$ molecule, and is on the order of several tenths $e$ per

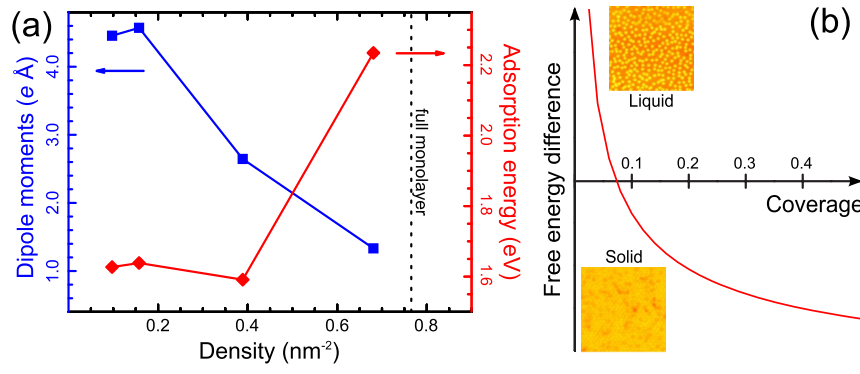

FIG. 5. Theoretical models to understand the surface phase transformation. (a) DFT calculations reveal that the dipole moments per molecule of $\mathrm{C}_{60} \mathrm{~F}_{48}$ on $\mathrm{SL}-\mathrm{WSe}_{2}$ (blue curve) decrease with the increasing molecular density, while the adsorption energy per molecule is in contrast (red curve). (b) A schematic diagram shows that the liquid phase is preferred at low coverage while the solid one is more favorable at high coverage.

molecule for all the densities considered here. Interestingly, the charge transfer as well as the dipole moment per molecule decrease with increasing molecular density. As shown in Fig. 5(a), when the molecular density is $0.1 \mathrm{~nm}^{-2}$ (close to the liquid phase), the $\mathrm{C}_{60} \mathrm{~F}_{48}$ dipole is as high as $4.4 e \AA$, which is comparable to the value of $3.6 e \AA$ derived from Fig. 4. As the density increases to $0.67 \mathrm{~nm}^{-2}$ (close to the fully close-packed monolayer), $\mu$ reduces significantly to $1.4 e \AA$ per molecule. The larger molecular dipole (charge transfer) per molecule at lower density is reasonable, because each molecule probably possesses larger electrostatic interactions with the substrate when it solely occupies a larger substrate area. In addition, compared to the $\mathrm{C}_{60} \mathrm{~F}_{48}$ /graphite interface, $\mathrm{C}_{60} \mathrm{~F}_{48} / \mathrm{WSe}_{2}$ /graphite has a relative larger molecular dipole (Fig. S12(d) of the Supplemental Material [49]). This enhancement can be explained as the molecule-graphite separation is increased by the $\mathrm{SL}-\mathrm{WSe}_{2}$ interlayer, and thus the interfacial dipole is magnified as the electron accumulation and depletion regions are mainly located in the molecule and graphite, respectively [31]. This is quite consistent with our experimental fitting that gives the value of the derived dipole for the $\mathrm{C}_{60} \mathrm{~F}_{48} /$ graphite as $2.5 e \AA$ (Fig. S11), which is slightly smaller. Consequently, the intermolecular separation (peak position) for the liquid $\mathrm{C}_{60} \mathrm{~F}_{48}$ on graphite surface is also smaller (Figs. S4 and S11) indicating a higher molecular density due to the weaker intermolecular repulsive interaction.

The adsorption energy per $\mathrm{C}_{60} \mathrm{~F}_{48}$ molecule on SL-WSe is calculated and shown as a red curve. It is found that the adsorption energy is $\sim 1.6 \mathrm{eV}$ when the density is lower than $0.4 \mathrm{~nm}^{-2}$, and increases to $2.25 \mathrm{eV}$ at $0.67 \mathrm{~nm}^{-2}$ (close packing). Therefore, the molecules are less stable at sparse liquid phases although the intermolecular dipole-dipole interactions are important; the molecules have higher stability at the close-packed solid phase because the attractive vdW forces become dominant.

Finally, the phase transformation can be understood from the perspective of the thermodynamic free energy for open systems. The free energy difference $\Delta \varepsilon$ between the 2D solid and liquid surface phases is a function of the molecular coverage $\sigma$, which can be written as (the derivation is given in 
the Supplemental Material [49])

$$
\Delta \varepsilon \propto \Delta \gamma+\frac{\alpha}{\sqrt{\sigma}}
$$

where $\Delta \gamma$ is the formation energy difference of the solid phase and liquid phase on the surface, and $\alpha>0$ is a parameter relative to the edge formation energy (see the Supplemental Material [49]). As obtained from DFT calculations $\gamma_{\text {liquid }}>$ $\gamma_{\text {solid }}$ and hence $\Delta \gamma<0$. Therefore, $\Delta \varepsilon$ has a characteristic curve shown in Fig. 5(b), as a reciprocal of the square root of $\sigma$. When $\Delta \varepsilon>0$, the liquid phase is more energetically stable and thereby is preferred; otherwise the solid phase is favored. Combined with STM results, the schematic diagram is plotted in Fig. 5(b), demonstrating that the liquid phase is observed at low $\sigma$ (i.e., $<0.1 \mathrm{ML}$ ), and the solid phase is obtained at high $\sigma$, as a result of the competition between multiple surface and interface interactions.

\section{CONCLUSION}

In summary, we have demonstrated a liquid-solid surface phase transformation of $\mathrm{C}_{60} \mathrm{~F}_{48}$ on a SL-WSe 2 surface investigated by LT-STM. When the surface coverage of $\mathrm{C}_{60} \mathrm{~F}_{48}$ molecules increases from $\sim 0.01 \mathrm{ML}$ to $0.5 \mathrm{ML}$, the packing conformations transform from small molecular clusters to liquid loose-packed islands with large intermolecular separations of several $\mathrm{nm}$, and finally to a solid close-packed structure. Statistical analysis of $\mathrm{C}_{60} \mathrm{~F}_{48}$ intermolecular distances and energy potentials reveals that the repulsive dipole-dipole interactions induced by interfacial charge transfer play important roles in the formation of $\mathrm{C}_{60} \mathrm{~F}_{48}$ liquid phases, as supported by first-principles calculations. More interestingly, the dipole moment per $\mathrm{C}_{60} \mathrm{~F}_{48}$ molecule varies with the surface molecule density. Therefore, the phase transformation is a result of the subtle competition between vdW forces and multiple substratemediated interactions at different coverages, which is more complicated than expected. The present study provides insights into the understanding of the growth behavior of organic molecules assembling on 2D TMD surfaces, which is important for the fabrication of organic-2D hybrid heterojunctions in future flexible electronic devices.

\section{ACKNOWLEDGMENTS}

A.T.S.W. acknowledges financial support from MOE AcRF Tier 1 Grant No. R-144-000-321-112 and the Graphene Research Centre. Y.L.H. and D.C. acknowledge the A-STAR SERC grant support for the 2D Growth Project under the 2D Pharos Program (SERC 1527000012). Calculations were performed on the Graphene Research Centre cluster supported by Prof. Su Ying Quek.
[1] D. Jariwala, T. J. Marks, and M. C. Hersam, Mixed-dimensional van der Waals heterostructures, Nat. Mater. 16, 170 (2017).

[2] D. B. Velusamy, M. A. Haque, M. R. Parida, F. Zhang, T. Wu, O. F. Mohammed, and H. N. Alshareef, 2D organic-inorganic hybrid thin films for flexible UV-visible photodetectors, Adv. Funct. Mater. 27, 1605554 (2017).

[3] A. T. S. Wee, M. C. Hersam, M. Chhowalla, and Y. Gogotsi, An update from Flatland, ACS Nano 10, 8121 (2016).

[4] W. H. Lee, J. Park, S. H. Sim, S. Lim, K. S. Kim, B. H. Hong, and K. Cho, Surface-directed molecular assembly of pentacene on monolayer graphene for high-performance organic transistors, J. Am. Chem. Soc. 133, 4447 (2011).

[5] S. J. Kang, G.-H. Lee, Y.-J. Yu, Y. Zhao, B. Kim, K. Watanabe, T. Taniguchi, J. Hone, P. Kim, and C. Nuckolls, Organic field effect transistors based on graphene and hexagonal boron nitride heterostructures, Adv. Funct. Mater. 24, 5157 (2014).

[6] S. Wieghold, J. Li, P. Simon, M. Krause, Y. Avlasevich, C. Li, J. A. Garrido, U. Heiz, P. Samorì, K. Müllen, F. Esch, J. V. Barth, and C. A. Palma, Photoresponse of supramolecular self-assembled networks on graphene-diamond interfaces, Nat. Commun. 7, 10700 (2016).

[7] X. Liu, Z. Wei, I. Balla, A. J. Mannix, N. P. Guisinger, E. Luijten, and M. C. Hersam, Self-assembly of electronically abrupt borophene/organic lateral heterostructures, Sci. Adv. 3, e1602356 (2017).

[8] D. Jariwala, S. L. Howell, K. S. Chen, J. M. Kang, V. K. Sangwan, S. A. Filippone, R. Turrisi, T. J. Marks, L. J. Lauhon, and M. C. Hersam, Hybrid, gate-tunable, van der Waals p-n heterojunctions from pentacene and $\mathrm{MoS}_{2}$, Nano Lett. 16, 497 (2016).

[9] F. Liu, W. L. Chow, X. He, P. Hu, S. Zheng, X. Wang, J. Zhou, Q. Fu, W. Fu, P. Yu, Q. Zeng, H. J. Fan, B. K. Tay, C. Kloc, and
Z. Liu, Van der Waals p-n junction based on an organic-inorganic heterostructure, Adv. Funct. Mater. 25, 5865 (2015).

[10] S. Vélez, D. Ciudad, J. Island, M. Buscema, O. Txoperena, S. Parui, G. A. Steele, F. Casanova, H. S. van der Zant, A. Castellanos-Gomez, and L. E. Hueso, Gate-tunable diode and photovoltaic effect in an organic-2D layered material $p-n$ junction, Nanoscale 7, 15442 (2015).

[11] C. E. Petoukhoff, M. B. Krishna, D. Voiry, I. Bozkurt, S. Deckoff-Jones, M. Chhowalla, D. M. O'Carroll, and K. M. Dani, Ultrafast charge transfer and enhanced absorption in $\mathrm{MoS}_{2}$-organic van der Waals heterojunctions using plasmonic metasurfaces, ACS Nano 10, 9899 (2016).

[12] C. Christodoulou, A. Giannakopoulos, G. Ligorio, M. Oehzelt, M. Timpel, J. Niederhausen, L. Pasquali, A. Giglia, K. Parvez, K. Mullen, D. Beljonne, N. Koch, and M. V. Nardi, Tuning the electronic structure of graphene by molecular dopants: Impact of the substrate, ACS Appl. Mater. Interfaces 7, 19134 (2015).

[13] J. Choi, H. Zhang, and J. H. Choi, Modulating optoelectronic properties of two-dimensional transition metal dichalcogenide semiconductors by photoinduced charge transfer, ACS Nano 10, 1671 (2016).

[14] Y. Huang, W. Zheng, Y. Qiu, and P. Hu, Effects of organic molecules with different structures and absorption bandwidth on modulating photoresponse of $\mathrm{MoS}_{2}$ photodetector, ACS Appl. Mater. Interfaces 8, 23362 (2016).

[15] H.-Z. Tsai, A. A. Omrani, S. Coh, H. Oh, S. Wickenburg, Y.-W. Son, D. Wong, A. Riss, H. S. Jung, G. D. Nguyen, G. F. Rodgers, A. Aikawa, T. Taniguchi, K. Watanabe, A. Zettl, S. G. Louie, J. Lu, M. L. Cohen, and M. F. Crommie, Molecular self-assembly in a poorly screened environment: $\mathrm{F}_{4} \mathrm{TCNQ}$ on graphene/BN, ACS Nano 9, 12168 (2015). 
[16] X. Lin, J. C. Lu, Y. Shao, Y. Y. Zhang, X. Wu, J. B. Pan, L. Gao, S. Y. Zhu, K. Qian, Y. F. Zhang, D. L. Bao, L. F. Li, Y. Q. Wang, Z. L. Liu, J. T. Sun, T. Lei, C. Liu, J. O. Wang, K. Ibrahim, D. N. Leonard, W. Zhou, H. M. Guo, Y. L. Wang, S. X. Du, S. T. Pantelides, and H. J. Gao, Intrinsically patterned two-dimensional materials for selective adsorption of molecules and nanoclusters, Nat. Mater. 16, 717 (2017).

[17] A. Wäckerlin, S. Fatayer, T. Nijs, S. Nowakowska, S. F. Mousavi, O. Popova, A. Ahsan, T. A. Jung, and C. Wäckerlin, Molecular chessboard assemblies sorted by site-specific interactions of outof-plane $d$-orbitals with a semimetal template, Nano Lett. 17, 1956 (2017).

[18] H. Huang, S. L. Wong, J. Sun, W. Chen, and A. T. S. Wee, Trapping single polar molecules in $\mathrm{SiC}$ nanomesh via out-ofplane dipoles, ACS Nano 6, 2774 (2012).

[19] M. Garnica, D. Stradi, S. Barja, F. Calleja, C. Diaz, M. Alcami, N. Martin, A. L. Vazquez de Parga, F. Martin, and R. Miranda, Long-range magnetic order in a purely organic 2D layer adsorbed on epitaxial graphene, Nat. Phys. 9, 368 (2013).

[20] C.-L. Song, B. Sun, Y.-L. Wang, Y.-P. Jiang, L. Wang, K. He, X. Chen, P. Zhang, X.-C. Ma, and Q.-K. Xue, Charge-TransferInduced Cesium Superlattices on Graphene, Phys. Rev. Lett. 108, 156803 (2012).

[21] G. Tomba, M. Stengel, W.-D. Schneider, A. Baldereschi, and A. De Vita, Supramolecular self-assembly driven by electrostatic repulsion: The 1D aggregation of rubrene pentagons on $\mathrm{Au}(111)$, ACS Nano 4, 7545 (2010).

[22] F. Bischoff, K. Seufert, W. Auwärter, S. Joshi, S. Vijayaraghavan, D. Écija, K. Diller, A. C. Papageorgiou, S. Fischer, F. Allegretti, D. A. Duncan, F. Klappenberger, F. Blobner, R. Han, and J. V. Barth, How surface bonding and repulsive interactions cause phase transformations: Ordering of a prototype macrocyclic compound on $\operatorname{Ag}(111)$, ACS Nano 7, 3139 (2013).

[23] R. Schiavilla, R. B. Wiringa, S. C. Pieper, and J. Carlson, Tensor Forces and the Ground-State Structure of Nuclei, Phys. Rev. Lett. 98, 132501 (2007).

[24] J. Fraxedas, S. García-Gil, S. Monturet, N. Lorente, I. FernándezTorrente, K. J. Franke, J. I. Pascual, A. Vollmer, R. P. Blum, N. Koch, and P. Ordejón, Modulation of surface charge transfer through competing long-range repulsive versus short-range attractive interactions, J. Phys. Chem. C 115, 18640 (2011).

[25] C. Stadler, S. Hansen, I. Kröger, C. Kumpf, and E. Umbach, Tuning intermolecular interaction in long-range-ordered submonolayer organic films, Nat. Phys. 5, 153 (2009).

[26] T. Aruga and Y. Murata, Alkali-metal adsorption on metals, Prog. Surf. Sci. 31, 61 (1989).

[27] R. Diehldag and R. McGrath, Current progress in understanding alkali metal adsorption on metal surfaces, J. Phys.: Condens. Matter 9, 951 (1997).

[28] F. Huttmann, A. J. Martínez-Galera, V. Caciuc, N. Atodiresei, S. Schumacher, S. Standop, I. Hamada, T. O. Wehling, S. Blügel, and T. Michely, Tuning the van der Waals Interaction of Graphene with Molecules via Doping, Phys. Rev. Lett. 115, 236101 (2015).

[29] C. Coletti, C. Riedl, D. S. Lee, B. Krauss, L. Patthey, K. von Klitzing, J. H. Smet, and U. Starke, Charge neutrality and bandgap tuning of epitaxial graphene on $\mathrm{SiC}$ by molecular doping, Phys. Rev. B 81, 235401 (2010).

[30] Y. J. Zheng, Y. L. Huang, Y. Chen, W. Zhao, G. Eda, C. D. Spataru, W. Zhang, Y.-H. Chang, L.-J. Li, D. Chi, S. Y. Quek, and
A. T. S. Wee, Heterointerface screening effects between organic monolayers and monolayer transition metal dichalcogenides, ACS Nano 10, 2476 (2016).

[31] Z. Song, T. Schultz, Z. Ding, B. Lei, C. Han, P. Amsalem, T. Lin, D. Chi, S. L. Wong, Y. J. Zheng, M.-Y. Li, L.-J. Li, W. Chen, N. Koch, Y. L. Huang, and A. T. S. Wee, Electronic properties of a 1D intrinsic/p-doped heterojunction in a 2D transition metal dichalcogenide semiconductor, ACS Nano 11, 9128 (2017).

[32] Y. Smets, C. B. Stark, S. Lach, F. Schmitt, C. A. Wright, M. Wanke, L. Ley, C. Ziegler, and C. I. Pakes, Charge-induced distortion and stabilization of surface transfer doped porphyrin films, J. Chem. Phys. 139, 044703 (2013).

[33] Advanced Fluoride-Based Materials for Energy Conversion, edited by T. Nakajima and H. Groult (Elsevier, Amsterdam, 2015).

[34] M. Dubois, K. Guérin, W. Zhang, Y. Ahmad, A. Hamwi, Z. Fawal, H. Kharbache, and F. Masin, Tuning the discharge potential of fluorinated carbon used as electrode in primary lithium battery, Electrochim. Acta 59, 485 (2012).

[35] P. Strobel, M. Riedel, J. Ristein, and L. Ley, Surface transfer doping of diamond, Nature (London) 430, 439 (2004).

[36] S. J. Sque, R. Jones, J. P. Goss, P. R. Briddon, and S. Öberg, First-principles study of $\mathrm{C}_{60}$ and $\mathrm{C}_{60} \mathrm{~F}_{36}$ as transfer dopants for p-type diamond, J. Phys.: Condens. Matter 17, L21 (2005).

[37] S. J. Sque, R. Jones, S. Öberg, and P. R. Briddon, Transfer doping of diamond: The use of $\mathrm{C}_{60}$ and $\mathrm{C}_{60} \mathrm{~F}_{36}$ to effect p-type surface conductivity, Phys. B (Amsterdam, Neth.) 376-377, 268 (2006).

[38] E. T. Mickelson, R. H. Hauge, and J. L. Margrave, Methylated and phenylated $\mathrm{C}_{60}$ from fluorinated fullerene precursors, J. Fluor. Chem. 92, 59 (1998).

[39] F. Okino, S. Yajima, S. Suganuma, R. Mitsumoto, K. Seki, and $\mathrm{H}$. Touhara, Fluorination of fullerene $\mathrm{C}_{60}$ and electrochemical properties of $\mathrm{C}_{60} \mathrm{~F}_{X}$, Synth. Met. 70, 1447 (1995).

[40] Y. L. Huang, Z. Ding, W. Zhang, Y. H. Chang, Y. Shi, L.-J. Li, Z. Song, Y. J. Zheng, D. Chi, S. Y. Quek, and A. T. S. Wee, Gap states at low-angle grain boundaries in monolayer tungsten diselenide, Nano Lett. 16, 3682 (2016).

[41] Y. Zhang, M. M. Ugeda, C. Jin, S. F. Shi, A. J. Bradley, A. Martin-Recio, H. Ryu, J. Kim, S. Tang, Y. Kim, B. Zhou, C. Hwang, Y. Chen, F. Wang, M. F. Crommie, Z. Hussain, Z. X. Shen, and S. K. Mo, Electronic structure, surface doping, and optical response in epitaxial $\mathrm{WSe}_{2}$ thin films, Nano Lett. 16 , 2485 (2016).

[42] J.-K. Huang, J. Pu, C.-L. Hsu, M.-H. Chiu, Z.-Y. Juang, Y.-H. Chang, W.-H. Chang, Y. Iwasa, T. Takenobu, and L.-J. Li, Largearea synthesis of highly crystalline $\mathrm{WSe}_{2}$ monolayers and device applications, ACS Nano 8, 923 (2014).

[43] G. Kresse and J. Hafner, Ab initio molecular dynamics for liquid metals, Phys. Rev. B 47, 558 (1993).

[44] D. Vanderbilt, Soft self-consistent pseudopotentials in a generalized eigenvalue formalism, Phys. Rev. B 41, 7892 (1990).

[45] P. E. Blöchl, Projector augmented-wave method, Phys. Rev. B 50, 17953 (1994).

[46] J. P. Perdew, K. Burke, and M. Ernzerhof, Generalized Gradient Approximation Made Simple, Phys. Rev. Lett. 77, 3865 (1996).

[47] M. Dion, H. Rydberg, E. Schröder, D. C. Langreth, and B. I. Lundqvist, Van der Waals Density Functional for General Geometries, Phys. Rev. Lett. 92, 246401 (2004). 
[48] J. Klimeš, D. R. Bowler, and A. Michaelides, Chemical accuracy for the van der Waals density functional, J. Phys.: Condens. Matter 22, 022201 (2010).

[49] See Supplemental Material at http://link.aps.org/supplemental/ 10.1103/PhysRevB.97.134102 for (1) adsorption of C60F48 molecules on SL-WSe2/graphite surface; (2) method used to extract the precise molecule position; (3) the phase transformation of C60F48 molecules on bare graphite surface; (4) recalibration of the molecular positions and intermolecular distances; (5) quasiparticle interference in $\mathrm{WSe}_{2}$ monolayer; (6) methods used to evaluate the intermolecular potential energy for $\mathrm{C} 60 \mathrm{~F} 48$ molecules; and (7) DFT calculations of the charge transfer, dipole moment, and binding energy of C60F48 on different substrates.

[50] S. Kawasaki, T. Aketa, H. Touhara, F. Okino, O. V. Boltalina, I. V. Gol'dt, S. I. Troyanov, and R. Taylor, Crystal structures of the fluorinated fullerenes $\mathrm{C}_{60} \mathrm{~F}_{36}$ and $\mathrm{C}_{60} \mathrm{~F}_{48}$, J. Phys. Chem. B 103, 1223 (1999).

[51] I. Horcas, R. Fernández, J. M. Gómez-Rodríguez, J. Colchero, J. Gómez-Herrero, and A. M. Baro, WSxM: A software for scanning probe microscopy and a tool for nanotechnology, Rev. Sci. Instrum. 78, 013705 (2007).

[52] T. Yokoyama, T. Takahashi, K. Shinozaki, and M. Okamoto, Quantitative Analysis of Long-Range Interactions between Adsorbed Dipolar Molecules on $\mathrm{Cu}(111)$, Phys. Rev. Lett. 98, 206102 (2007).

[53] D. Chandler, J. D. Weeks, and H. C. Andersen, Van der Waals picture of liquids, solids, and phase transformations, Science 220, 787 (1983).

[54] S. C. B. Mannsfeld and T. Fritz, Analysis of the substrate influence on the ordering of epitaxial molecular layers: The special case of point-on-line coincidence, Phys. Rev. B 69, 075416 (2004).

[55] S. C. B. Mannsfeld and T. Fritz, Understanding organicinorganic heteroepitaxial growth of molecules on crystalline substrates: Experiment and theory, Phys. Rev. B 71, 235405 (2005).

[56] M. Gruenewald, C. Sauer, J. Peuker, M. Meissner, F. Sojka, A. Schöll, F. Reinert, R. Forker, and T. Fritz, Commensurism at electronically weakly interacting phthalocyanine/PTCDA heterointerfaces, Phys. Rev. B 91, 155432 (2015).

[57] J. Renard, M. B. Lundeberg, J. A. Folk, and Y. Pennec, Real-Time Imaging of K Atoms on Graphite: Interactions and Diffusion, Phys. Rev. Lett. 106, 156101 (2011).
[58] M. Caragiu and S. Finberg, Alkali metal adsorption on graphite: A review, J. Phys.: Condens. Matter 17, R995 (2005).

[59] N. Knorr, H. Brune, M. Epple, A. Hirstein, M. A. Schneider, and K. Kern, Long-range adsorbate interactions mediated by a two-dimensional electron gas, Phys. Rev. B 65, 115420 (2002).

[60] J. Repp, F. Moresco, G. Meyer, K.-H. Rieder, P. Hyldgaard, and M. Persson, Substrate Mediated Long-Range Oscillatory Interaction between Adatoms: $\mathrm{Cu} / \mathrm{Cu}(111)$, Phys. Rev. Lett. 85, 2981 (2000).

[61] P. Han, and P. S. Weiss, Electronic substrate-mediated interactions, Surf. Sci. Rep. 67, 19 (2012).

[62] L. Petersen, P. Laitenberger, E. Lægsgaard, and F. Besenbacher, Screening waves from steps and defects on $\mathrm{Cu}(111)$ and $\mathrm{Au}(111)$ imaged with STM: Contribution from bulk electrons, Phys. Rev. B 58, 7361 (1998).

[63] L. Chen, P. Cheng, and K. Wu, Quasiparticle interference in unconventional 2D systems, J. Phys.: Condens. Matter 29, 103001 (2017).

[64] P. Mallet, I. Brihuega, S. Bose, M. M. Ugeda, J. M. GómezRodríguez, K. Kern, and J. Y. Veuillen, Role of pseudospin in quasiparticle interferences in epitaxial graphene probed by high-resolution scanning tunneling microscopy, Phys. Rev. B 86, 045444 (2012).

[65] I. Brihuega, P. Mallet, C. Bena, S. Bose, C. Michaelis, L. Vitali, F. Varchon, L. Magaud, K. Kern, and J. Y. Veuillen, Quasiparticle Chirality in Epitaxial Graphene Probed at the Nanometer Scale, Phys. Rev. Lett. 101, 206802 (2008).

[66] G. M. Rutter, J. N. Crain, N. P. Guisinger, T. Li, P. N. First, and J. A. Stroscio, Scattering and interference in epitaxial graphene, Science 317, 219 (2007).

[67] M. Yankowitz, D. McKenzie, and B. J. LeRoy, Local Spectroscopic Characterization of Spin and Layer Polarization in $\mathrm{WSe}_{2}$, Phys. Rev. Lett. 115, 136803 (2015).

[68] H. Liu, J. Chen, H. Yu, F. Yang, L. Jiao, G.-B. Liu, W. Ho, C. Gao, J. Jia, W. Yao, and M. Xie, Interference in epitaxial monolayer tungsten diselenide, Nat. Commun. 6, 8180 (2015).

[69] P. Hyldgaard and M. Persson, Long-ranged adsorbate-adsorbate interactions mediated by a surface-state band, J. Phys.: Condens. Matter 12, L13 (2000).

[70] Y. Pan, S. Fölsch, Y. Nie, D. Waters, Y. Lin, B. Jariwala, K. Zhang, K. Cho, J. A. Robinson, and R. M. Feenstra, Quantumconfined electronic states arising from the moiré pattern of $\mathrm{MoS}_{2}-\mathrm{WSe}_{2}$ heterobilayers, Nano Lett. 18, 1849 (2018). 